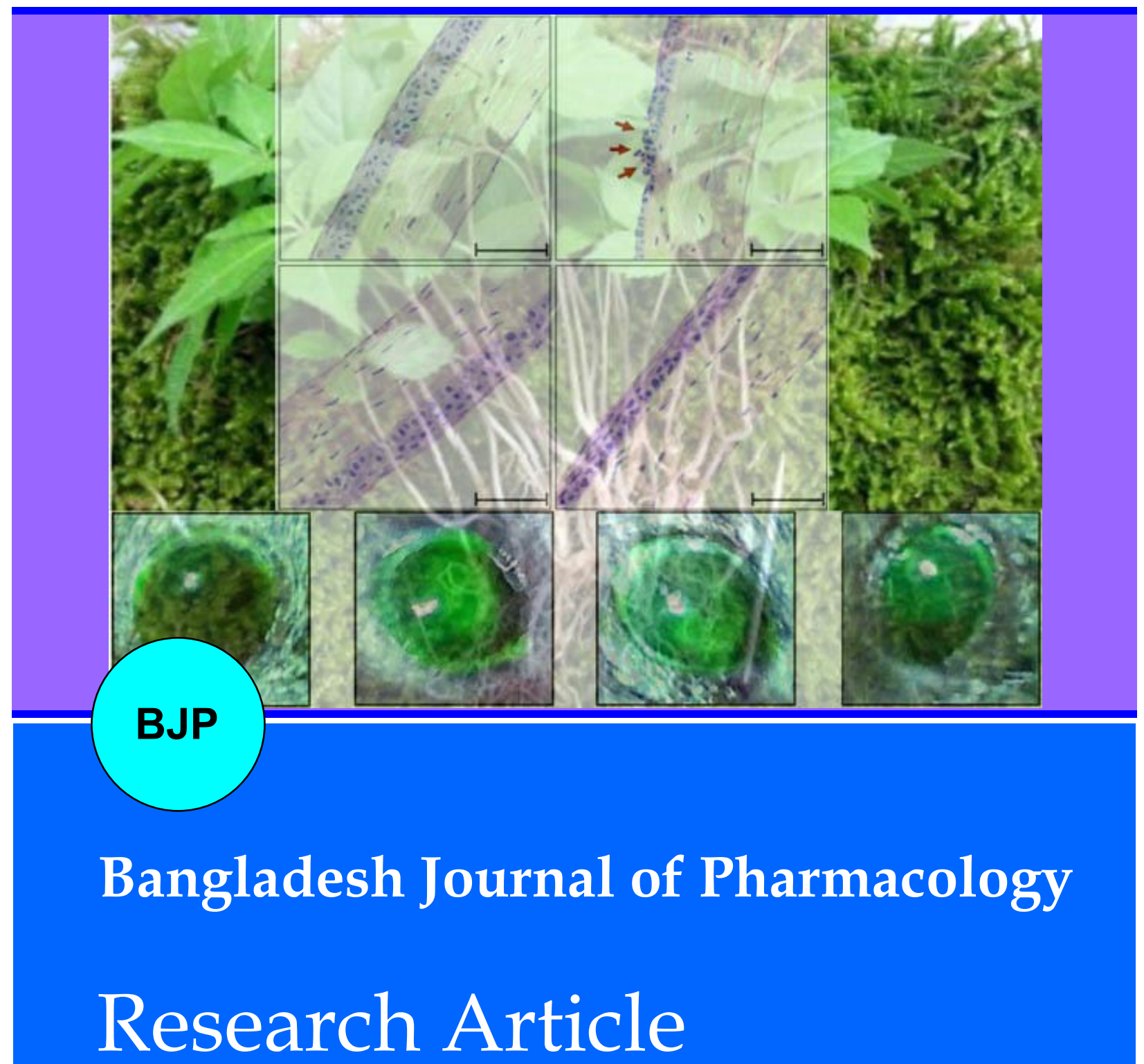

Amelioration of dry eye syndrome by oral administration of cultivated wild ginseng extract 


\title{
Amelioration of dry eye syndrome by oral administration of cultivated wild ginseng extract
}

\author{
Jae Sung Pyo', Hyung Hoi Kim², Kang Min Kim³ and Jae Seon Kang1 \\ ${ }^{1}$ Department of Pharmacy, Kyungsung University, Busan 48434, Korea; ${ }^{2}$ Department of Laboratory Medicine and \\ Biomedical Research Institute, Pusan National University Hospital, Pusan 50612, Korea; ${ }^{3}$ Department of \\ Pharmaceutical Science and Technology, Kyungsung University, Busan 48434, Korea.
}

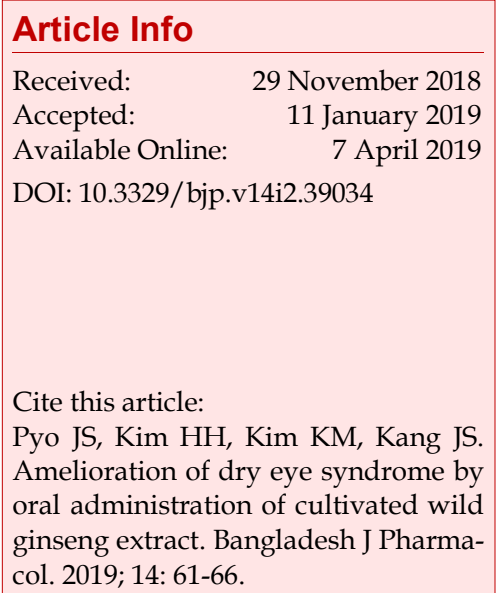

\begin{abstract}
Cultivated wild ginseng extract has been reported to have chemopreventive, chemotherapeutic, hepatoprotective, cardioprotective and anti-inflammatory effects. We investigated the preventive effect of ginseng extract (oral administration, 0.5 or $1.0 \mathrm{mg} / \mathrm{kg}$ twice daily for 2 weeks) on dry eye syndrome using benzalkonium chloride-induced dry eye mouse model. Corneal epithelial cells and tear volume were measured using fluorescein corneal staining, followed by histologic assessment, TUNEL assay and Western blot analysis for TNF-a expression. Tear volume was restored to control level with lower fluorescein corneal staining score. Structural damage by benzalkonium chloride of corneal epithelial cells was significantly inhibited in the histologic assessment. Both TUNEL assay and TNF-a expression level showed that apoptotic-positive cells were significantly decreased. These findings indicate that ginseng extract could be a promising supplement in dry eye syndrome treatment.
\end{abstract}

\section{Introduction}

Dry eye syndrome (keratoconjunctivitis sicca) is a multifactorial disease of ocular surface accompanying visual disturbance, hyperemia, photophobia and ocular discomfort (Lemp et al., 2007). Its pathogenesis is triggered by dysfunction of the lacrimal functional unit leading towards an alteration in tear quantity and composition, which results in damage of the conjunctival and corneal epithelium with accompanying inflammatory events (Messmer et al., 2010; Javadi and Feizi, 2011; Tsubota et al., 2017). Accordingly, drugs contain-ing anti-inflammatory agents, especially steroid eye drop, has been the most common and effective treatment of dry eye syndrome. However, non-steroidal anti-inflammatory drugs (NSAIDs) are increasingly being demanded as alternative to steroidal drug, in order to avoid its potential adverse effects, such as glaucoma (Colligris et al., 2014; Phulke et al., 2017).
Ginsenosides, which are the active components of the herb ginseng, have been regarded as main active ingredients for the pharmaceutical activities of ginseng roots (Yun, 2003). Previous reports showed that ginsenoside possess remarkable pharmaceutical activities in cancer (Kang et al., 2009), anti-inflammatory (Wang et al., 2012), anti-allergic (Bae et al., 2002) and anti-aging effect (Kang et al., 2009). Ginsenoside compound K (20O-beta-D-glucopyranosyl-20(S)-protopanaxadiol) is one of the most biologically and pharmaceutically active protopanaxadiol (PPD)-type ginsenoside (Shin et al., 2017). It is noteworthy that compound $K$ has antiinflammatory effect through suppression of inflammation related-genes (Wang et al., 2016), indicating that compound $\mathrm{K}$ could be promising drug candidate for dry eye syndrome.

In the present study, the protective effect of cultivated wild ginseng extract using benzalkonium chloride- 
induced dry eye mouse was examined.

\section{Materials and Methods}

\section{Preparation and analysis of ginseng extract}

Cultivated wild ginseng was fully dried at $121^{\circ} \mathrm{C}$ for 15 min, followed by grinding a total of $60 \mathrm{~g}$. The ground ginseng was extracted 5 times with $300 \mathrm{~mL}$ of $>85 \%$ ethanol. After centrifugation at 10,000 rpm for $10 \mathrm{~min}$, the supernatant was harvested and evaporated at $50^{\circ} \mathrm{C}$ using rotary evaporator. The extracted saponin (10 g) was re-suspended in water $(1,000 \mathrm{~mL})$ to form a bulk active protopanaxadiol. The suspension material was prepared in an active form (compound K) component in order to decompose the sugar side chains of the saponin by a pectinase enzyme solution $(170 \mathrm{~mL})$ using a peristaltic pump $(50 \mathrm{~mL} / \mathrm{min})$ and exposed overnight at $4{ }^{\circ} \mathrm{C}$. Compound $\mathrm{K}$ was collected by centrifugation at $10,000 \mathrm{rpm}$ for $10 \mathrm{~min}$, supernatant were re-suspended in alcohol $(200 \mathrm{~mL})$ and filtrated. The filtrated material was evaporated and used in this study. Cultivated wild ginseng extracts were obtained and analyzed as previously described (Ok et al., 2016).

\section{Animal experiment}

Male C57BL/6 ICR mice (7 weeks old) were obtained from the Hyochang Science, Korea. In order to minimize environmental changes, the mice were housed for one week. The mice were divided into control group, positive group, and two treatment groups (four mice in each group). The control group was treated with $100 \mu \mathrm{L}$ saline orally at 10:00 AM for 2 weeks, followed by saline intraocularly $(5 \mu \mathrm{L})$ in the eyes at 2:00 PM for 2 weeks (control group). The positive control group was administered orally $200 \mu \mathrm{L}$ solvent once per day at 10:00 AM, then $5 \mu \mathrm{L}$ benzalkonium chloride eye drops at 2:00 PM for 2 weeks. The two treatment groups

\section{Box 1: Phenol Red Thread Test}

\section{Principle}

When a cotton thread impregnated with phenol red touches the tear (slightly alkaline), the thread turns from yellow to red. Then the length of red color in $\mathrm{mm}$ is recorded.

Uses

This test is used to detect the dry eye syndrome

\section{Requirements}

Ketamine; Phenol red thread (Tiankin Jingming New Technological Development Co., Ltd., China); Pilocarpine; Xylazine

\section{Procedure}

Step 1: Each mouse was anesthetized by ketamine $(60 \mathrm{mg} / \mathrm{kg}$ body weight) and xylazine $(6 \mathrm{mg} / \mathrm{kg})$

Step 2: Pilocarpine $(2.5 \mathrm{mg} / \mathrm{kg})$ was injected intraperitoneally received ginseng extract orally (either $100 \mu \mathrm{L}$ of $0.5 \mathrm{mg} /$ $\mathrm{kg}$ or $1 \mathrm{mg} / \mathrm{kg}$, respectively) at 10:00 AM, followed by receiving $5 \mu \mathrm{L}$ benzalkonium chloride eye drops at 2:00 PM for 2 weeks.

\section{Ocular surfaces evaluation with fluorescein staining}

The corneal epithelial surface was assessed with fluorescein staining (Zhang et al., 2014). One microliter of $0.1 \%$ sodium fluorescein was dropped into the right eye. After $1 \mathrm{~min}$, the corneal epithelial surface was observed with a cobalt blue filter under a slit-lamp microscope (DM6C ophthalmoscope, Zumax Medical Co. Ltd., Germany). Fluorescein corneal staining scores (ranging between 1 and 4) from all groups were determined by four independent observers and then averaged. The cornea was divided into four quadrants, which were scored respectively. Staining scores were determined as follows: 1; staining absent, 2; sparse punctuate staining less than 10 spots, 3; approximately one-third confluency of punctuate staining, 4; nearly half confluency of punctuate staining. The scores from four quadrants were summed to determine grade (total, 16 points). The analysis was performed in a doubleblind manner.

\section{Histopathology}

The hematoxylin and eosin staining were used to measure benzalkonium chloride-induced damage of corneal epithelial cells. The eyeball was fixed in 10\% formalin solution. Then the cornea was isolated, following by dehydration and paraffin embedding. The cornea $(4 \mu \mathrm{m})$ was cut from the paraffin blocks and were stained with $0.1 \%$ hematoxylin and $1 \%$ eosin. Each section was observed under a light microscope (DP-70, Olympus, Japan).

\section{TUNEL assay}

TUNEL assays were carried out to observe apoptotic

\section{to stimulate the tear secretion}

Step 3: The phenol red thread was placed under the lateral one fifth of the inferior palpebral margin

Step 4: The thread absorbed the tear that contact it for $5 \mathrm{~min}(0$ $-5 \mathrm{~min}$ and $5-10 \mathrm{~min}$ )

\section{Calculation}

The tear volume was gauged by the length of the phenol red thread left in contact with the eye

\section{Notes}

1. Less time is required for each eye

2. Thread is difficult to handle initially due to its light and flexible nature

\section{References}

Arakaki et al., 2014; Senchyna and Wax, 2008 
cells in corneal epithelial layer. The formalin embedded eyeball sections were deparaffinized and rehydrated sections $(5 \mu \mathrm{m})$. Then obtained sections were placed in $3 \%$ hydrogen peroxide for $10 \mathrm{~min}$ at room temperature, and treated with $20 \mu \mathrm{g} / \mathrm{mL}$ proteinase $\mathrm{K}$ for $10 \mathrm{~min}$ at $37^{\circ} \mathrm{C}$. After washing three times in phosphate buffered saline, apoptotic cells were stained using commercial kit (In situ cell death detection kit, Roche, Germany) according to the supplier's instructions.

\section{Western blot analysis}

After TUNEL staining observation, cryosections were used for TNF-a expression using Western blotting analysis. Cells were lysed and extracted in RIPA buffer (50 mM Tris-Cl [pH 7.6], $150 \mathrm{mM} \mathrm{NaCl}, 1 \%$ Triton X-100, $1 \%$ NP-40, $1 \%$ sodium deoxycholate, $0.1 \%$ sodium dodecyl sulfate, $1 \mathrm{mM}$ EDTA). The supernatants were harvested by $14,000 \mathrm{rpm}$ centrifugation at $4^{\circ} \mathrm{C}$ for 15 min. Total proteins $(30 \mu \mathrm{g})$ were separated using $15 \%$ sodium dodecyl sulfate polyacrylamide gel (SDSPAGE). Nonspecific binding was blocked by TBST (25 $\mathrm{mM}$ Tris- $\mathrm{HCl}, 50 \mathrm{mM} \mathrm{NaCl}, 0.05 \%$ Tween-20) containing $5 \%$ dry milk for 1 hour at room temperature, followed by incubation $4^{\circ} \mathrm{C}$ for 16 hours with $1: 400$ and $1: 10,000$ diluted primary antibodies of anti-TNF-a and anti- $\beta$-actin, respectively. After 1 hour of incubation at room temperature with appropriate secondary antibody, the signal was detected by the ChemiDoc-It2 Imaging system (UVP 97-0650-05) using enhanced chemiluminescent solutions (ECL Substrate, Thermo Scientific, USA). The relative densities were determined using ImageJ software (version 1.51j8; public domain program created by Wayne Rasband, National Institutes of Health, USA).

\section{Statistical analysis}

The data is expressed as the mean \pm standard deviation. Statistical analyses were performed and analyzed by one-way analysis of variance $(n=4)$. $p$ value below 0.01 was considered significant.

\section{Results}

The contents of cultivated wild ginseng extract (active saponin) by HPLC had a result of a compound $\mathrm{K}$ of $52 \%$, $\mathrm{Rd}$ of $34.4 \%, \mathrm{~F} 2$ of $2.69 \%$, and $\mathrm{Rg} 3$ of $2 \%$ with respect to the total saponin content.

\section{Amount of tear in dry eye mouse model}

Figure 1 shows that there was a significant decrease (approximately $25.7 \%, \mathrm{p}<0.01$ ) of tear volume in $0.2 \%$ benzalkonium chloride-induced mice (positive control group), which confirms that dry eye condition was successfully induced. No significant difference was observed in the ginseng extract $(0.5 \mathrm{mg} / \mathrm{kg})$-treated group compared to the positive control group.
Remarkably, decreased tear volume in ginseng extract $(1 \mathrm{mg} / \mathrm{kg})$-treated group were restored to more than $95 \%$ of the control $(\mathrm{p}<0.001)$. There was statistically significant differences between the positive control group and the $1 \mathrm{mg} / \mathrm{kg}$-treated group with respect to the protective effects of ginseng extract.

\section{Clinical evaluations of dry eye}

After 2 weeks of oral administration of ginseng extract, fluorescein staining was carried out for clinical evaluations on corneal surface of dry eye mouse (Figure

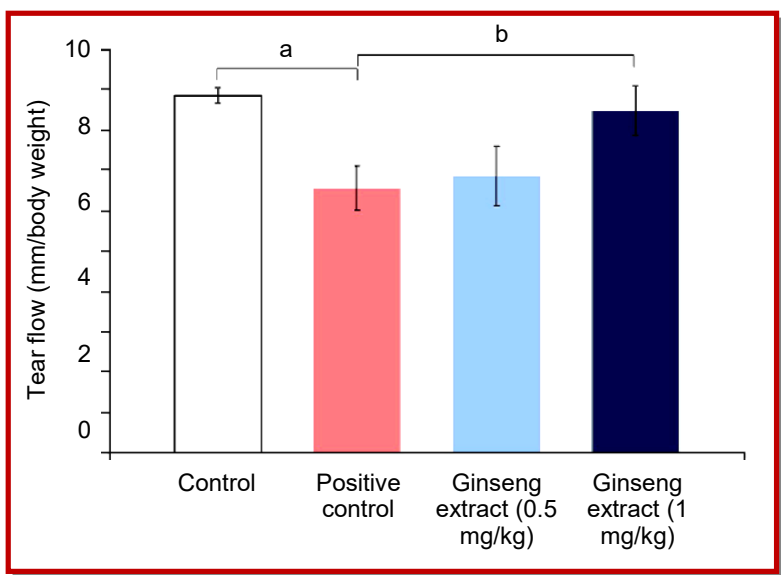

Figure 1: Effects of ginseng extract $(0.5$ and $1 \mathrm{mg} / \mathrm{kg}$ ) administration on tear secretion in benzalkonium chloride-induced dry eye syndrome mouse model. Results are expressed as mean \pm SD; $\left(n=4\right.$, a $\left.<<0.01,{ }^{b} p<0.001\right)$

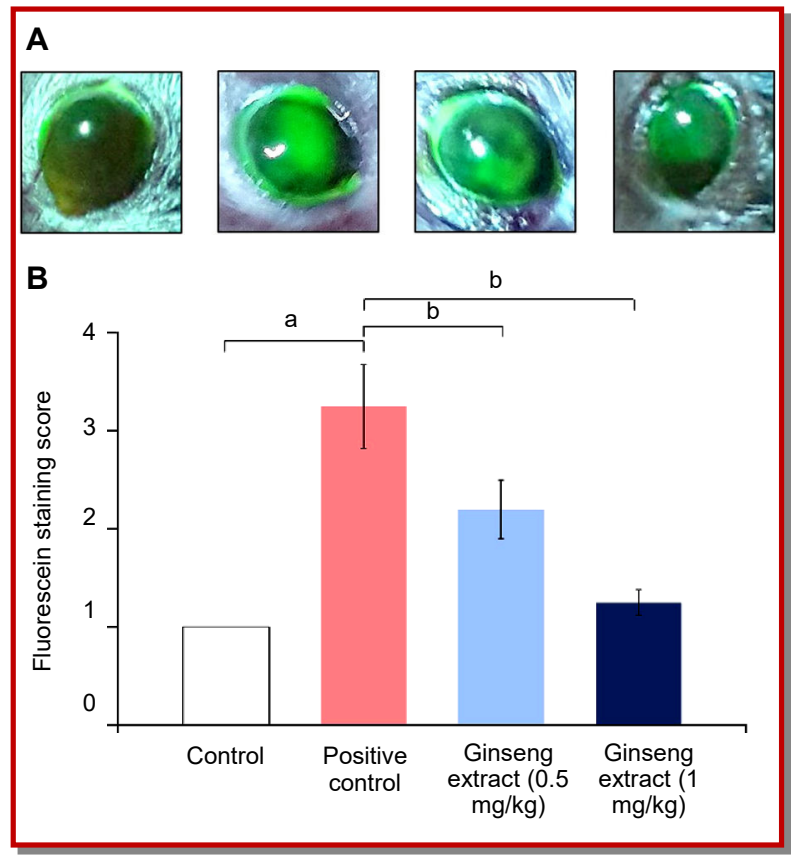

Figure 2: Clinical evaluation of dry eye including representative images of corneal fluorescein staining (A) and staining scores (B) after 14 days and in the normal control. Results are expressed as mean \pm SD for 4 mice per group ( ${ }^{a} \mathrm{p}<0.01$, $\left.{ }^{b} \mathrm{p}<0.001\right)$ 
2A). There was a remarkable increase of fluorescein staining scores in positive control group (mean score: $3.4, \mathrm{p}<0.01$ ), compared to the normal group (mean score: $1.9,1.1$, respectively, Figure $2 \mathrm{~B}$ ). It is noteworthy that fluorescein staining scores were dramatically decreased in benzalkonium chloride-treated group, compared to the positive control group $(\mathrm{p}<0.001)$.

\section{Structural damage of corneal epithelial cells}

After 2 weeks of treatment, damaged cells with irregular and vacuolated shapes were observed in the corneas of positive control group (benzalkonium chloride $0.2 \%$ treated) as shown in Figure 3B compared to normal group (Figure 3A). It should be noted that benzalkonium chloride-induced damages of the superficial

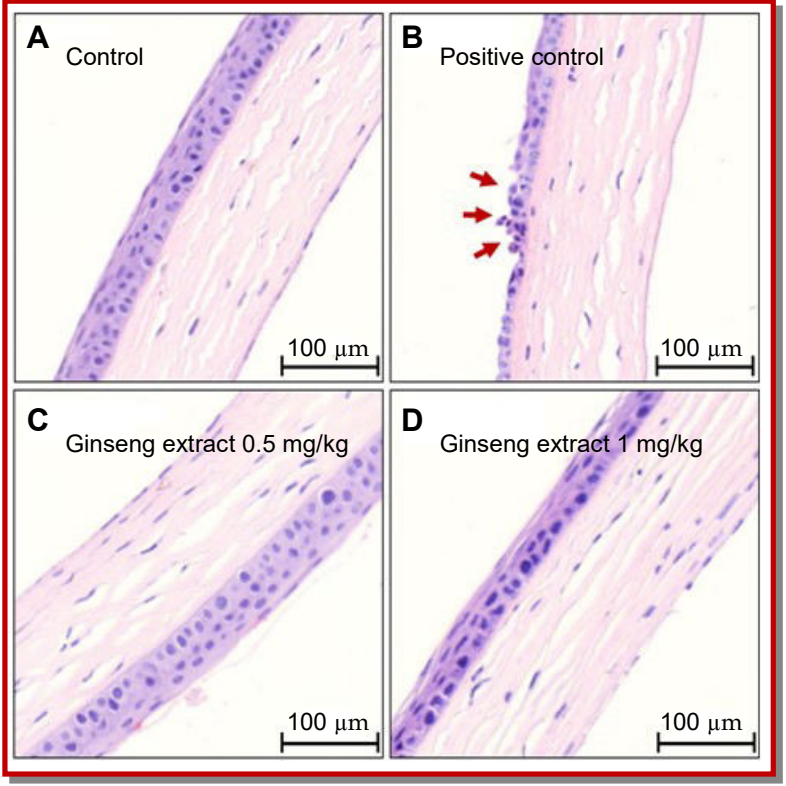

Figure 3: Effect of ginseng extract on corneal epithelial cells of benzalkonium chloride-induced mice. Red arrows indicates irregular and vacuolated corneal epithelial cells epithelium in the corneas were remarkably recovered to the morphology of the normal group, in both ginseng extract-treated groups (Figure 3C and 3D), which indicates the protective role of ginseng extract in dry eye.

\section{Effect of ginseng extract on apoptosis in corneal epithelial cells}

In order to investigate the preventive role of ginseng extract against benzalkonium chloride-induced apoptosis, TUNEL assay was performed (Figure 4A). Figure 4B shows that significant increase (3.6-fold, $\mathrm{p}<0.01$ ) of TUNEL positive cells in positive control group in comparison to control group. There was considerable reduction of TUNEL positive cells in ginseng extract-treated group, compared to positive control group $(\mathrm{p}<0.01)$. Interestingly, the proportion of apoptotic cells were also decreased with the increase of ginseng extract concentration, which demonstrates antiapoptotic activity of ginseng extract.

To further examine the anti-apoptotic effect of ginseng extract, expression level of TNF-a was assessed by Western blotting. Compared with control group, TNF-a expression was enhanced in positive control group (Figure 5A). Remarkably, TNF-a induction by benzalkonium chloride treatment was significantly suppressed in ginseng extract $0.5 \mathrm{mg} / \mathrm{kg}$ treated group by $52.6 \%$ (Figure 5B). Most importantly, it should be noted that expression of TNF-a was completely abolished.

\section{Discussion}

In present study, we examined the ginseng extract as nutritional supplement for amelioration of dry eye mouse syndrome model. Interestingly, ginseng extract restored the decreased amount of tear when compared to control group. Furthermore, two weeks treatment of ginseng extract remarkably recovered the damages in

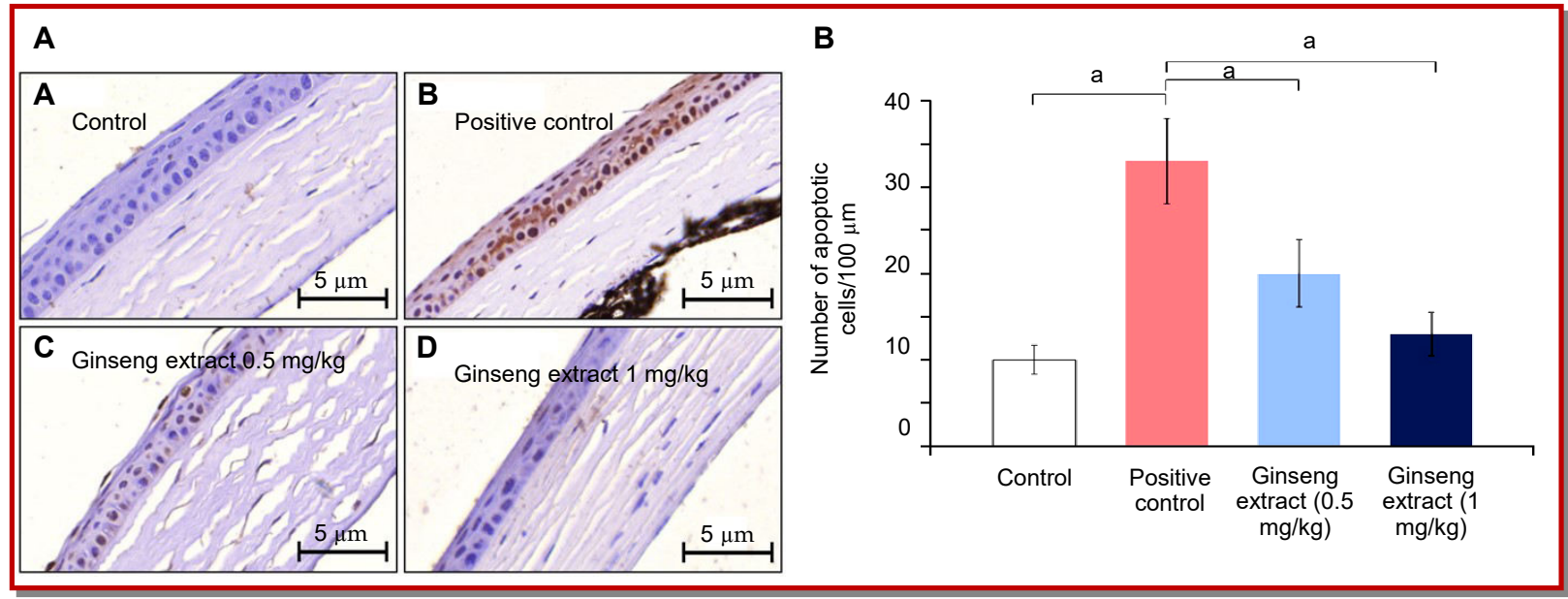

Figure 4: Evaluation of apoptosis via TUNEL assay on corneal sections. (A) Representative TUNEL staining images of corneal epithelial cells. (B) The number of apoptotic (TUNEL-positive) cells per $100 \mu \mathrm{m}$ tissue. Results are expressed as means \pm SD for 4 mice per group. $(\mathrm{n}=4, \mathrm{p}<0.01)$ 

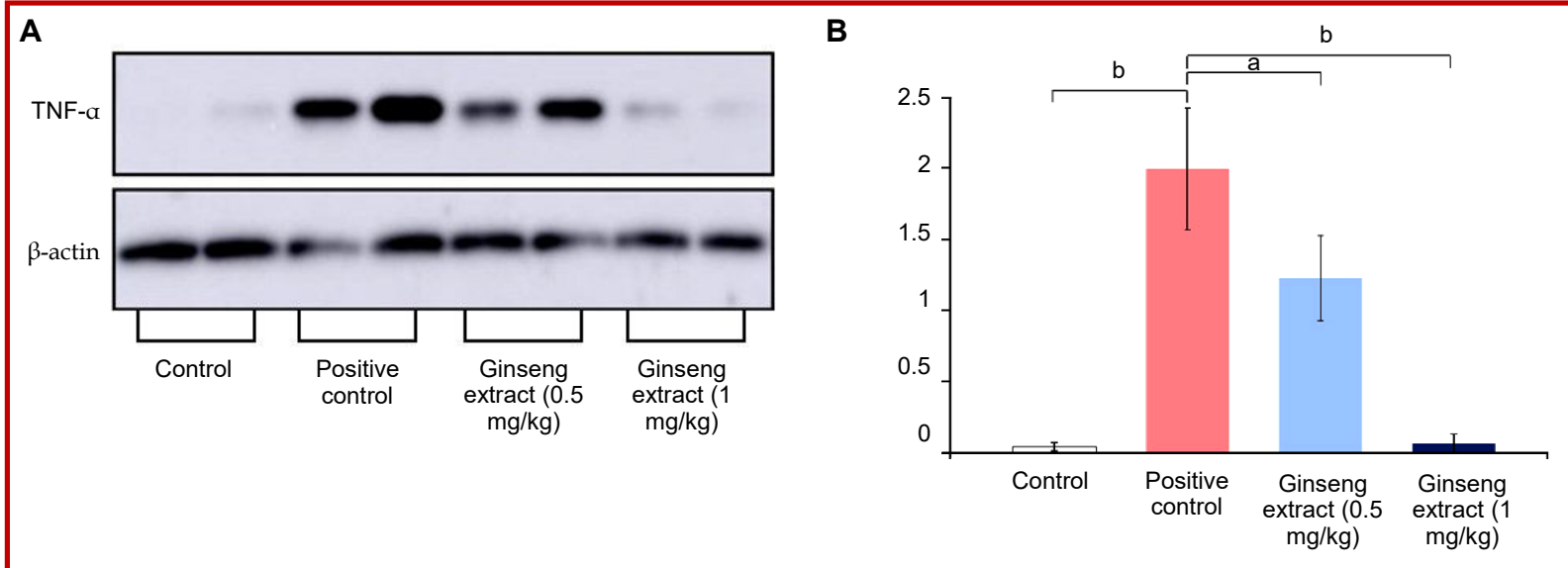

Figure 5: Effect of ginseng extract on TNF-a expression in cornea tissue of benzalkonium chloride-induced dry eye syndrome mouse. (A) Western-blot analysis for TNF-a. (B) The expression of TNF- $\alpha$ was gauaged by normalization based on $\beta$-actin intensity as shown in (A). The expression value of the control was set to 1.0. $\left(n=4,{ }^{a} p<0.05,{ }^{b} p<0.01\right)$

the superficial epithelium of corneas to the normal morphology. In parallel, ginseng extract reduced the apoptotic cells with accompanying suppression of TNFa expression, which is crucial in pro-inflammatory reactions. These results demonstrated that oral administration of ginseng extract significantly mitigated the benzalkonium chloride-induced damage of mouse corneal epithelial cells via suppression of inflammation, which indicating that ginseng extract could be a prospective supplement for dry eye syndrome.

The final concentrate is, hereinafter, referred to as gingseng extract because the PPD-based active ingredients such as compound $\mathrm{K}, \mathrm{Rd}, \mathrm{F} 2$, and $\mathrm{Rg} 3$ occupy $91 \%$ and the content of the compound $\mathrm{K}$ is highest as $52 \%$. Ginsenoside metabolite compound $\mathrm{K}$ such as active saponin is a kind of dammarane-type triterpene ginseng saponins. There have been many studies demonstrating anti-inflammatory activity of compound K (Cuong et al., 2009; Joh et al., 2011). Wang et al., 2012 reported compound K could reduce mRNA expression of inflammatory-related genes including tumor necrosis factor (TNF), inter-leukin 1 (IL-1), interleukin 4 (IL-4), interferon $\mathrm{g}$ (INFG), and prostaglandin-endoperoxide synthase 2 (PTGS2). Furthermore, Wu et al., 2014 revealed that compound $\mathrm{K}$ inhibited the activation of mitogen-activated protein kinases (MAPKs) and Toll-like receptors 4 (TLR4)/ lipopolysaccharide-induced nuclear factor $\mathrm{kB}$, with accompanying reduced-expression of pro-inflammatory cytokines secreted by macrophages (Kim et al., 2017). There is another evidence that compound $\mathrm{K}$ remarkably inhibited superoxide generation, NADPH oxidase activities, and Ser345-p47phox phosphorylation in macrophages (Cuong et al., 2009). Recent studies using in vivo animal models shows that ginsenosides metabolites including compound $\mathrm{K}$ exert anti-inflammatory activities in several inflammatory diseases models as well (Kim et al., 2017). It is noteworthy that Korean red ginseng significantly improved the tear film stability and total ocular surface disease index score, as compared to placebo (Kim et al., 2010).

Consistent with this result, ginseng has been suggested as prospective drug candidate for glaucoma or cataract based on the anti-apoptotic/anti-oxidative activity of ginsenosides (Kim et al., 2010; Lee et al., 2010; Huynh et al., 2013)

\section{Conclusion}

Ginseng extract significantly inhibited inflammation in dry eye syndrome mouse model by suppression of TNF -a expression. It could be promising supplement for dry eye syndrome treatment.

\section{Ethical Issue}

The animal experiment in present study was carried out in strict accordance to the World Health Organization guideline, and the Institutional Review Board of Kyungsung University for the evaluation of the safety and efficacy of herbal medicines (confirmation number: Research-17-013A).

\section{Conflict of Interest}

The authors declare no conflict of interest.

\section{Acknowledgement}

This research was supported by a Kyungsung University Research Grants in 2018.

\section{References}

Arakaki R, Eguchi H, Yamada A, Kudo Y, Iwasa A, Enkhmaa T, Hotta F, Mitamura-Aizawa S, Mitamura Y, Hayashi Y, 
Ishimaru N. Anti-inflammatory effects of rebamipide eye drop administration on ocular lesions in a murine model of primary Sjogren's syndrome. PLoS One. 2014; 9: e98390.

Bae EA, Choo MK, Park EK, Park SY, Shin HY, Kim DH. Metabolism of ginsenoside $\mathrm{R}(\mathrm{c})$ by human intestinal bacteria and its related antiallergic activity. Biol Pharm Bull. 2002; 25: 743-47.

Colligris B, Alkozi HA, Pintor J. Recent developments on dry eye disease treatment compounds. Saudi J Ophthalmol. 2014; 28: $19-30$.

Cuong TT, Yang CS, Yuk JM, Lee HM, Ko SR, Cho BG, Jo EK. Glucocorticoid receptor agonist compound $\mathrm{K}$ regulates dectin-1-dependent inflammatory signaling through inhibition of reactive oxygen species. Life Sci. 2009; 85: 625-33.

Huynh TP, Mann SN, Mandal NY. Botanical compounds: Effects on major eye diseases. Evid Based Complement Alternat Med. 2013; 2013: 549174.

Javadi MA, Feizi S. Dry eye syndrome. J Ophthalmic Vis Res. 2011; 6: 192-98.

Joh EH, Lee IA, Jung IH, Kim DH. Ginsenoside Rb1 and its metabolite compound $\mathrm{K}$ inhibit IRAK-1 activation--the key step of inflammation. Biochem Pharmacol. 2011; 82: 278-86.

Kang TH, Park HM, Kim YB, Kim H, Kim N, Do JH, Kang C, Cho Y, Kim SY. Effects of red ginseng extract on UVB irradiation-induced skin aging in hairless mice. J Ethnopharmacol. 2009; 123: 446-51.

Kim JH, Yi YS, Kim MY, Cho JY. Role of ginsenosides, the main active components of Panax ginseng, in inflammatory responses and diseases. J Ginseng Res. 2017; 41: 435-43.

Kim NR, Kim JH, Kim CY. Effect of Korean red ginseng supplementation on ocular blood flow in patients with glaucoma. J Ginseng Res. 2010; 34: 237-45.

Lee SM, Sun JM, Jeong JH, Kim MK, Wee WR, Park JH, Lee JH. Analysis of the effective fraction of sun ginseng extract in selenite induced cataract rat model. J Korean Ophthalmol Soc. 2010; 51: 733-39.

Lemp MA, Baudouin C, Baum J, Dogru M, Foulks GN, Kinoshita S, Laibson P, McCulley J, Murube J, Pflugfelder SC, Rolando M. The definition and classification of dry eye disease: Report of the definition and classification subcommittee of the international dry eye workshop. Ocul Surf. 2007; 5: 75-92.

Messmer EM, Bulgen M, Kampik A. Hyperosmolarity of the tear film in dry eye syndrome. Dev Ophthalmol. 2010; 45: 129-38.
Ok S, Kang JS, Kim KM. Simultaneous analysis method for polar and non-polar ginsenosides in cultivated wild ginseng by reversed-phase HPLC-CAD. J Life Sci. 2016; 26: 247-52.

Phulke S, Kaushik S, Kaur S, Pandav SS. Steroid-induced glaucoma: An avoidable irreversible blindness. J Curr Glaucoma Pract. 2017; 11: 67-72.

Senchyna M, Wax MB. Quantitative assessment of tear production: A review of methods and utility in dry eye drug discovery. J Ocul Biol Dis Infor. 2008; 1: 1-6.

Shin KC, Choi HY, Seo MJ, Oh DK. Improved conversion of ginsenoside $\mathrm{Rb} 1$ to compound $\mathrm{K}$ by semi-rational design of Sulfolobus solfataricus beta-glycosidase. AMB Express. 2017; 7: 186 .

Tsubota K, Yokoi N, Shimazaki J, Watanabe H, Dogru M, Yamada M, Kinoshita S, Kim HM, Tchah HW, Hyon JY, Yoon KC, Seo KY, Sun X, Chen W, Liang L, Li M, Liu Z. New Perspectives on dry eye definition and diagnosis: A consensus report by the asia dry eye society. Ocul Surf. 2017; 15: 65-76.

Wang CZ, Du GJ, Zhang Z, Wen XD, Calway T, Zhen Z, Musch MW, Bissonnette M, Chang EB, Yuan CS. Ginsenoside compound $\mathrm{K}$, not $\mathrm{Rb1}$, possesses potential chemopreventive activities in human colorectal cancer. Int J Oncol. 2012; 40: 1970-76.

Wang L, Zhang Y, Chen J, Li S, Wang Y, Hu L, Wu Y. Immunosuppressive effects of ginsenoside-Rd on skin allograft rejection in rats. J Surg Res. 2012; 176: 267-74.

Wang Y, Chen J, Luo X, Zhang Y, Si M, Wu H, Yan C, Wei W. Ginsenoside metabolite compound $\mathrm{K}$ exerts joint-protective effect by interfering with synoviocyte function mediated by TNF-alpha and tumor necrosis factor receptor type 2. Eur J Pharmacol. 2016; 771: 48-55.

Wu H, Chen J, Wang Q, Jia X, Song S, Yuan P, Liu K, Liu L, Zhang Y, Zhou A, Wei W. Ginsenoside metabolite compound $\mathrm{K}$ attenuates inflammatory responses of adjuvant -induced arthritis rats. Immunopharmacol Immunotoxicol. 2014; 36: 124-29.

Yun TK. Experimental and epidemiological evidence on nonorgan specific cancer preventive effect of Korean ginseng and identification of active compounds. Mutat Res. 2003; 523 -24: 63-74.

Zhang Z, Yang WZ, Zhu ZZ, Hu QQ, Chen YF, He H, Chen YX, Liu ZG. Therapeutic effects of topical doxycycline in a benzalkonium chloride-induced mouse dry eye model. Invest Ophthalmol Vis Sci. 2014; 55: 2963-74. 